\title{
ON THE KAROUBI FILTRATION OF A CATEGORY
}

\author{
MANUEL CÁRDENAS AND ERIK KJÆR PEDERSEN
}

\section{INTRODUCTION}

In [5] Karoubi introduced the notion of an additive category $\mathcal{U}$ being filtered by a full subcategory $\mathcal{A}$ (The precise statement - definition 1.5 pages 115-116 of [5] is recalled in definition 3.2). He then used this to give an axiomatic description of negative K-groups, including an exact sequence

$$
K_{0}(\mathcal{A}) \rightarrow K_{0}(\mathcal{U}) \rightarrow K_{0}(\mathcal{U} / \mathcal{A}) \rightarrow K_{-1}(\mathcal{A}) \rightarrow \ldots
$$

This sequence was generalized to hold for higher K-groups in [7] where a fibration of spectra was obtained

$$
K\left(\mathcal{A}^{\wedge K}\right) \rightarrow K(\mathcal{U}) \rightarrow K(\mathcal{U} / \mathcal{A}) .
$$

Here $\mathcal{A}^{\wedge K}$ is a certain subcategory of the idempotent completion of $\mathcal{A}$. In particular $K(\mathcal{A})$ and $K\left(\mathcal{A}^{\wedge K}\right)$ only differ at $K_{0}$. This fibration was generalized in [3] to produce a fibration

$$
K^{-\infty}(\mathcal{A}) \rightarrow K^{-\infty}(\mathcal{U}) \rightarrow K^{-\infty}(\mathcal{U} / \mathcal{A})
$$

Where $K^{-\infty}$ is a non-connective spectrum whose negative homotopy groups are the negative $K$-groups of $\mathcal{A}$, and whose connective cover is the usual $K$-theory spectrum. These fibrations have been applied to produce excision results in controlled algebraic $K$-theory, see [1], [2], [3], [4]. Most of these excision results are easy consequences of the above fibrations. As an example we derive one of the excision results of [3] in the final section.

The proof in [7] was based on the double mapping cylinder construction of Thomason [10]. In recent years a number of results in algebraic $K$-theory have been given easier proofs by using Waldhausen's S.-construction see e. g. [6], and [9]. In this paper we give a proofs of 1.0.1 using that method. The basic idea is to consider finite chain complexes in $\mathcal{U}$ and two notions of weak equivalence, chain homotopy equivalence and chain maps inducing homotopy equivalence in $\mathcal{U} / \mathcal{A}$. The proof then is an

Research by the first author was supported in part by proyecto DGICYT PB92-0672

Research by the second author was supported in part by NSF DMS 9104026 
application of Waldhausen's generic fibration lemma [12, Theorem 1.6.4], and identification of the terms. This identification uses results of Thomason and Trobaugh [11], which we recall in section 6 . We also give a proof of 1.0.2 in the final section.

It is our aim to make this paper as self contained as possible. In the first sections we recall the basic notions and results we shall need in this paper.

\section{Categories With Cofibrations and Weak Equivalences}

In this section we present a quick review of Waldhausens $K$-theory of a small category with cofibrations and weak equivalences [12]. One example to keep in mind is an additive category where the cofibrations are inclusions of direct summands up to isomorphism, and the weak equivalences isomorphisms. Another example is finite chain complexes in an additive category with cofibrations the degreewise inclusions on direct summands and weak equivalences the homotopy equivalences. If we take chain complexes and weak equivalences only the isomorphisms, we have an example of an exact category ( exact sequences are only degreewise split exact). In addition we recall the basic tools which will allow us to decide when two categories have isomorphic $K$-theory.

Given any small category $\mathcal{C}$, satisfying certain properties described below Waldhausen assigns functorially to $\mathcal{C}$ a topological space $K(\mathcal{C})$, which we call $K$-theory of $\mathcal{C}$. The homotopy groups are defined to be the $K$-groups of $\mathcal{C}$. This extends the classical definitions of $K$-groups of a ring $R$ by taking $\mathcal{C}$ to be the additive category of finitely generated projective modules over $R$.

Definition 2.1. [12, Sections 1.1 and 1.2] A small category $\mathcal{C}$ with a zero object is said to be a category with cofibrations and weak equivalences if it has two distinguished subcategories, $c o \mathcal{C}$ and $w \mathcal{C}$, satisfying the following axioms:

a) $c o \mathcal{C}$ axioms.

cof 1 Isomorphisms in $\mathcal{C}$ are cofibrations.

$\overline{\operatorname{cof} 2}$ For every $A \in \mathcal{C}, * \rightarrow A$ is a cofibration.

$\overline{\operatorname{cof} 3}$ Cofibrations admit cobase change:

a: If $A \rightarrow B$ is a cofibration and $A \rightarrow C$ any map, then the push out exists in $\mathcal{C}$.

b: $C \rightarrow C \cup_{A} B$ is a cofibration.

b) $w \mathcal{C}$ axioms.

weq 1 Isomorphisms in $\mathcal{C}$ are weak equivalences.

$\overline{w e q 2}$ (Gluing Lemma) If in the commutative diagram

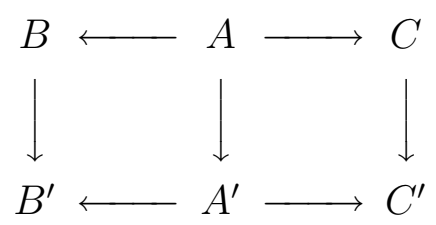


the horizontal arrows on the left are cofibrations and all three vertical arrows are in $w \mathcal{C}$ then

$$
B \bigcup_{A} C \rightarrow B^{\prime} \bigcup_{A} C
$$

is in $w \mathcal{C}$.

The two following axioms may, or may not, be satisfied by $\mathcal{C}$.

Saturation axiom: If $a, b$ are composable maps in $\mathcal{C}$ and if two of $a, b, a b$ are in $w \mathcal{C}$ then so is the third.

Extension axiom: Let

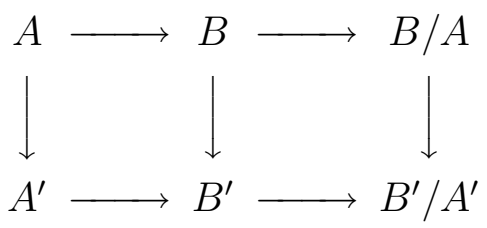

be a map of cofibrations sequences $\left(B / A=* \bigcup_{A} B\right)$. If $A \rightarrow A^{\prime}$ and $B / A \rightarrow B^{\prime} / A^{\prime}$ are in $w \mathcal{C}$ then $B \rightarrow B^{\prime}$ is in $w \mathcal{C}$ as well.

Having fixed $c o \mathcal{C}$ and $w \mathcal{C}$, we have a simplicial category:

$$
\begin{aligned}
\text { S.C }: \Delta^{o p} & \longrightarrow(c a t) \\
{[n] } & \longmapsto S_{n} \mathcal{C}
\end{aligned}
$$

where $S_{n} \mathcal{C}$ is the category of objects:

$$
* \rightarrow A_{1} \rightarrow A_{2} \rightarrow \cdots \rightarrow A_{n}
$$

with chosen quotients $A_{i, j}=A_{i} / A_{j}, 1 \leq i \leq j \leq n$ where $A_{i, i}=*$ always. The degeneraicy maps are given by inserting identities, and the boundary map $d_{i}$ by omitting the index $i$. This is almost like the nerve of the category, which since there is an initial object would give a contractible space. The difference lies in $d_{0}$, omitting the 0th index means taking all the quotients, hence the need for chosen quotients. It is a category with cofibrations and weak equivalences, by defining a map $A \rightarrow A^{\prime}$ to be a cofibration if

$$
A_{j} \longrightarrow A_{j}^{\prime} \quad \text { and } \quad A_{j}^{\prime} \bigcup_{A_{j}} A_{j+1} \longrightarrow A_{j+1}^{\prime}
$$

are cofibrations in $\mathcal{C}$ for all $j$. An arrow $A \rightarrow A^{\prime}$ is defined to be a weak equivalence if the arrow $A_{i, j} \longrightarrow A_{i, j}^{\prime}$ is a weak equivalence for each pair $i \leq j$. We thus have that $S$. is a functor from categories with cofibrations and weak equivalences to simplicial categories with cofibrations and weak equivalences. For more details about this see sections 1.1, 1.2 and 1.3 in [12].

We can think of: 


$$
\begin{aligned}
w S . \mathcal{C}: \Delta^{o p} & \longrightarrow(c a t) \\
{[n] } & \longmapsto w S_{n} \mathcal{C}
\end{aligned}
$$

as a bisimplicial set by taking the nerve of $w S_{n} \mathcal{C}$

Definition 2.2. [12, Section 1.3] The Algebraic K-theory of the category with cofibrations $\mathcal{C}$, with respect to the category of weak equivalences $w \mathcal{C}$ is given by the pointed space

$$
\Omega|w S \cdot \mathcal{C}|=K(\mathcal{C})
$$

The $K$-groups of $\mathrm{C}$ are the homotopy groups of $K(\mathcal{C})$

$$
K_{*} \mathcal{C}=\pi_{*}(\Omega|w S . \mathcal{C}|)\left(=\pi_{*+1}|w S . \mathcal{C}|\right) .
$$

Actually $K$-theory can be described as a spectrum rather than just a space. The S.-construction extends namely, by naturality, to simplicial categories with cofibrations and weak equivalences. In particular therefore it applies to S.C to produce a bisimplicial category with cofibrations and weak equivalences, S.S.C. Again the construction extends to bisimplicial categories with cofibrations and weak equivalences; and so on. Therefore we get a spectrum

$$
n \mapsto|w S . \cdots S . \mathcal{C}| .
$$

The structural maps are defined as the adjoint of the map $\Sigma|w \mathcal{C}| \rightarrow|w S . \mathcal{C}|$ which is given as the inclusion of the 1-skeleton in the $S$--construction, see [12, page 329].

It turns out that the spectrum is a $\Omega$-spectrum beyond the first term (the additivity theorem 2.7 below is needed to prove this). As the spectrum is connective (the $n$-th term is (n-1)-connected) an equivalent assertion is that in the sequence

$$
|w \mathcal{C}| \rightarrow \Omega|w S . \mathcal{C}| \rightarrow \Omega^{2}|w S . S . \mathcal{C}| \rightarrow \cdots
$$

all maps except the first are homotopy equivalences. Hence $K$-theory of $\mathcal{C}$ could equivalently be defined as the infinite loop space

$$
\Omega^{\infty}\left|w S \cdot{ }^{(\infty)} \mathcal{C}\right|=\lim _{n} \Omega^{n}\left|w S \cdot{ }^{(n)} \mathcal{C}\right|
$$

We will refer to either of the three versions as the $K$-theory of $\mathcal{C}$ and denote it as $K(\mathcal{C})$.

Now we recall criteria that determine when two categories have homotopy equivalent $K$-theories. Some extra structure is required on the category. It is necessary to have a notion of cylinder in order to define some kind of homotopy theory. 
Definition 2.3. A functor $F: C \rightarrow C^{\prime}$, between categories with cofibrations and weak equivalences is said to be exact if $F$ preserves all relevant structures. In a natural way such a functor induces a map

$$
w S . F: w S . \mathcal{C} \rightarrow w S . \mathcal{C}^{\prime}
$$

and therefore a map between the $K$-theories.

2.4. Because of the properties of the product and the realization functor, given a map (simplicial homotopy)

$$
H: X \times I \rightarrow Y
$$

where $X$ and $Y$ are simplicial sets, it induces a homotopy

$$
H:|X| \times I \rightarrow|Y|
$$

between $|F|=\left|H_{|X| \times\{0\}}\right|$ and $|G|=\left|H_{|X| \times\{1\}}\right|$. This applies, in particular, to our case when $X$ and $Y$ are the $S$.-constructions of categories $\mathcal{C}$ and $\mathcal{C}^{\prime}$.

Therefore we have an idea of homotopy between functors. To see more about this we refer the reader to [13, Section 5, Notions of homotopy theory].

Definition 2.5. [12, Section 1.6] A category $\mathcal{C}$ with cofibrations and weak equivalences has a cylinder functor if there is a functor

$$
T: A r C \rightarrow D i a g C
$$

where $A r \mathcal{C}$ is the category of arrows of $\mathcal{C}$ and DiagC is the category of diagrams in $\mathcal{C}$.

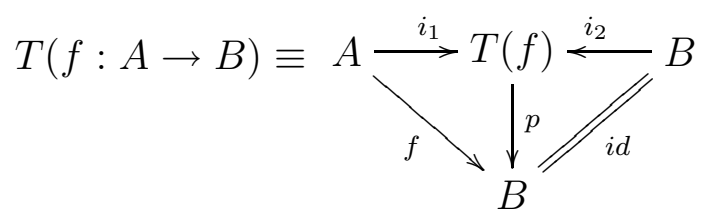

satisfying:

Cyl 1: Front and back inclusion assemble to an exact functor

$$
\begin{aligned}
A r \mathcal{C} & \rightarrow F_{1} \mathcal{C} \\
(f: A \rightarrow B) & \rightarrow(A \vee B>T(f))
\end{aligned}
$$

where $F_{1} \mathcal{C}$ is the full subcategory of $A r \mathcal{C}$ whose objects are the cofibrations in $\mathcal{C}$.

Cyl 2: $T(* \rightarrow A)=A$, for every $A \in C$ and projection and back inclusion are the identity on $A$.

There is an additional axiom that is often satisfied:

Cylinder axiom: The projection $T(f) \rightarrow B$ is in $w \mathcal{C}$ for every $f: A \rightarrow B$. 
Definition 2.6. A cofibration sequence of exact functors $\mathcal{C} \rightarrow \mathcal{C}^{\prime}$ is a sequence of natural transformations $F^{\prime} \rightarrow F \rightarrow F^{\prime \prime}$ having the property that for every $A \in \mathcal{C}$ $F^{\prime}(A) \rightarrow F(A) \rightarrow F^{\prime \prime}(A)$ is a cofibration sequence in $\mathcal{C}^{\prime}$.

One of the basic tools is the additivity theorem [12, Theorem 1.4.2 and Proposition 1.3.2], see also [6].

Theorem 2.7. There is a homotopy equivalence

$$
|w S . F| \cong\left|w S .\left(F^{\prime} \vee F^{\prime \prime}\right)\right|
$$

This statement is equivalent to:

(i) The map

$$
\begin{aligned}
w S . E(\mathcal{A}, \mathcal{C}, \mathcal{B}) & \longrightarrow w S . \mathcal{A} \times w S . \mathcal{B} \\
A \rightarrow C \rightarrow B & \longmapsto(A, B)
\end{aligned}
$$

is a homotopy equivalence.

(ii) The map

$$
\begin{aligned}
w S . E(\mathcal{C}) & \longrightarrow w S . \mathcal{C} \times w S . \mathcal{C} \\
A \rightarrow C \rightarrow B & \longmapsto(A, B)
\end{aligned}
$$

is a homotopy equivalence.

(iii) These two maps are homotopic

$$
\begin{gathered}
w S . E(\mathcal{C}) \longrightarrow w S . \mathcal{C} \\
A \rightarrow C \rightarrow B \longmapsto C, A \vee B
\end{gathered}
$$

Let's see how the $K$-theories of a category and a subcategory relate to each other.

Definition 2.8. Let $\mathcal{A}$ be an exact subcategory of the exact category $\mathcal{B}$. $\mathcal{A}$ is said to be cofinal in $\mathcal{B}$ if $0 \rightarrow A^{\prime} \rightarrow B \rightarrow A^{\prime \prime} \rightarrow 0$ is exact in $\mathcal{B}$ with $A^{\prime}$ and $A^{\prime \prime}$ are in $\mathcal{A}$, then so is $B$, and if for each $B$ in $\mathcal{B}$ there is a $B^{\prime}$ in $\mathcal{B}$ so that $B \oplus B^{\prime}$ is isomorphic to an object in $\mathcal{A}$. (For simplicity we will assume $\mathcal{A}$ is isomorphism closed in $\mathcal{B}$. This doesn't change the $K$-theory of $\mathcal{A}$ ).

The next theorem is known as the cofinality theorem.

Theorem 2.9. [9, Theorem 2.1]

Let $\mathcal{A}$ be cofinal in $\mathcal{B}$ and $G=K_{0}(\mathcal{B}) / K_{0}(\mathcal{A})$. Then there is a fibration sequence up to homotopy

$$
K(i S . \mathcal{A}) \rightarrow K(i S . \mathcal{B}) \rightarrow B G
$$

Notice $w \mathcal{A}=i \mathcal{A}$ and $w \mathcal{B}=i \mathcal{B}$, where $i$ denotes the isomorphisms, the minimal possible choice. 
In general, given a category $\mathcal{C}$ we will fix the cofibrations and then look at the interplay of the two $K$-theories defined by two differents notions of weak equivalences. Let $\mathcal{C}$ be a category with cofibrations equipped with two categories of weak equivalences, one finer than the other $v \mathcal{C} \subset w \mathcal{C}$. Let $\mathcal{C}^{w}$ denote the full subcategory with cofibrations of $\mathcal{C}$ given by the objects $A$ in $\mathcal{C}$ having the property $* \rightarrow A$ is in $w \mathcal{C}$. It inherits weak equivalences:

$$
v \mathcal{C}^{w}=\mathcal{C}^{w} \cap v \mathcal{C} \quad w \mathcal{C}^{w}=\mathcal{C}^{w} \cap w \mathcal{C}
$$

Now recall the generic fibration lemma.

Lemma 2.10. [12, Theorem 1.6.4]

If $\mathcal{C}$ has a cylinder functor, and the coarse category of weak equivalences $w \mathcal{C}$ satisfies the cylinder axiom, saturation axiom and extension axiom, then the square:

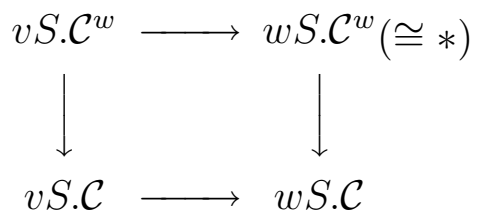

is homotopy cartesian, and the upper right term is contractible.

Next we recall the approximation theorem, a sufficient condition for an exact functor $F: \mathcal{A} \rightarrow \mathcal{B}$ to induce a homotopy equivalence $w S . \mathcal{A} \rightarrow w S . \mathcal{B}$.

Definition 2.11. Let $F: \mathcal{A} \rightarrow \mathcal{B}$ be an exact functor of categories with cofibrations and weak equivalences. We say it has the approximation property if it satisfies:

App 1: An arrow in $\mathcal{A}$ is a weak equivalence in $\mathcal{A}$ if and only if its image in $\mathcal{B}$ is a weak equivalence in $\mathcal{B}$.

$A p p$ 2: Given any object $A$ in $\mathcal{A}$ and any map $x: F(A) \rightarrow B$ in $\mathcal{B}$ there exists a cofibration $a: A \rightarrow A^{\prime}$ in $\mathcal{A}$ and a weak equivalence $x^{\prime}: F\left(A^{\prime}\right) \rightarrow B$ in $\mathcal{B}$ such that

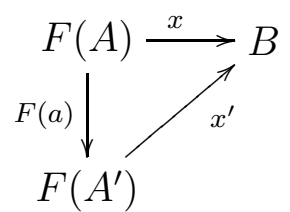

commutes.

The approximation theorem says:

Theorem 2.12. [12, Theorem 1.6.7]

Let $\mathcal{A}$ and $\mathcal{B}$ be categories with cofibrations and weak equivalences. Assume $w \mathcal{A}$ and $w \mathcal{B}$ satisfy the saturation axiom. Suppose $\mathcal{A}$ has a cylinder functor that satisfies the cylinder axiom. Let $F: \mathcal{A} \rightarrow \mathcal{B}$ be an exact functor having the approximation properties. Then $w \mathcal{A} \rightarrow w \mathcal{B}$ and $w S . \mathcal{A} \rightarrow w S . \mathcal{B}$ induce homotopy equivalences. 


\section{Additive Categories. Filtrations.}

In this and the next section, we recall Karoubi's notion of filtration of an additive category [5] and present the natural structures of categories of cofibrations and weak equivalences that this concepts lead to.

Definition 3.1. An additive category is a small category with a zero object 0 , where $\operatorname{Hom}(U, V)$, the group of morphisms between objects $U$ and $V$, are abelian. Moreover, the composition is bilinear with respect this operation. Finite product and coproducts exist in these categories and are isomorphic.

All definitions that follow in this section are taken from [7, Section 5].

Let $\mathcal{A}$ be a full subcategory of the additive category $\mathcal{U}$. We shall use the letters $A--F$ (resp. $U--Z)$ to denote objects of $\mathcal{A}$ (resp. $\mathcal{U})$.

Definition 3.2. We say $\mathcal{U}$ is $\mathcal{A}$-filtered if every object $U$ has a family of decompositions $\left\{U=E_{\alpha} \oplus U_{\alpha}\right\}$ (called a filtration of $U$ ) satisfying the following axioms:

F1: For Each $U$, the decompositions form a filtered poset under the partial order $E_{\alpha} \oplus U_{\alpha} \leq E_{\beta} \oplus U_{\beta}$ whenever $U_{\beta} \subset U_{\alpha}$ and $E_{\alpha} \subset E_{\beta}$.

F2: Every map $A \rightarrow U$ factors $A \rightarrow E_{\alpha} \rightarrow E_{\alpha} \oplus U_{\alpha}=U$ for some $\alpha$.

F3: Every map $U \rightarrow A$ factors $U=E_{\alpha} \oplus U_{\alpha} \rightarrow E_{\alpha} \rightarrow A$ for some $\alpha$.

F4: For each $U, V$ the filtration on $U \oplus V$ is equivalent to the sum of filtrations $\left\{U=E_{\alpha} \oplus U_{\alpha}\right\}$ and $\left\{V=F_{\beta} \oplus V_{\beta}\right\}$, i.e. to $\left\{U \oplus V=\left(E_{\alpha} \oplus F_{\beta}\right) \oplus\left(U_{\alpha} \oplus V_{\beta}\right)\right\}$.

Definition 3.3. We now suppose given an $\mathcal{A}$-filtered category $\mathcal{U}$. Call a map $U \rightarrow V$ completely continuous, $(c c)$, if it factors through an object in $\mathcal{A}$. $\mathcal{U} / \mathcal{A}$ is defined to be the category with the same objects as $\mathcal{U}$ but with

$$
\operatorname{Hom}_{\mathcal{U} / \mathcal{A}}(U, V)=\operatorname{Hom}_{\mathcal{U}}(U, V) /\{c c \text { maps }\}
$$

i.e. two maps are the same if their difference factors through an object in $\mathcal{A}$.

The additive categories $\mathcal{U}$ and $\mathcal{U} / \mathcal{A}$ have a natural structure as category of cofibrations and weak equivalences where cofibrations are the morphisms that are isomorphic to split monomorphisms into direct summands and weak equivalences are the isomorphisms.

Given the $\mathcal{A}$-filtration of $\mathcal{U}$ we can endow $\mathcal{U}$ with another, larger, category of weak equivalences than the isomorphisms of $\mathcal{U}$. This new one, $w$, will be those morphisms whose classes in $\mathcal{U} / \mathcal{A}$ are isomorphisms. We retain the same category of cofibrations as in $\mathcal{U}$. The category $\mathcal{U}$ with this choice of cofibrations and weak equivalences will be denoted $\mathcal{U}(\mathcal{A})$.

The objective is to apply the generic fibration lemma 2.10, to the identity functor

$$
\mathcal{U} \longrightarrow \mathcal{U}(\mathcal{A})
$$


hoping to obtaining as fiber the category $\mathcal{A}$. We can not use these categories directly since neither has cylinder functor as the generic fibration lemma requires. Therefore we need to consider the corresponding categories of finite chain complexes.

\section{The CATEgory $C(\mathcal{U})$ And its structures}

Given an additive category $\mathcal{U}$, we can define the category of finite chain complexes in $\mathcal{U}$, where objects are:

$$
C_{\#}: 0 \rightarrow C_{r} \stackrel{d}{\rightarrow} C_{r-1} \stackrel{d}{\rightarrow} \cdots \rightarrow C_{l} \rightarrow 0
$$

such that $d^{2}=0$, i.e. $d^{2}$ factors through the zero object. A chain map $f: C_{\#} \rightarrow D_{\#}$ is a collection of morphisms $f=\left\{f_{r}: C_{r} \rightarrow D_{r}\right\}$ such that $d_{D} f=f d_{C}$. A chain homotopy in $\mathcal{A}$

$$
e: f \simeq f^{\prime}: C \rightarrow D
$$

is a collection of morphisms $\left\{e: C_{r} \rightarrow D_{r+1}\right\}$ such that $d_{D} e+e d_{C}=f^{\prime}-f: C_{r} \rightarrow D_{r}$. A chain equivalence is a chain map $f: C \rightarrow D$ which admits a chain homotopy inverse, that is, a chain map $g: D \rightarrow C$ such that

$$
\exists h: g f \simeq 1: C \rightarrow C \text { and } k: f g \simeq 1: D \rightarrow D .
$$

The cofibrations will be those chain maps which degree-wise are inclusions into direct summands. The weak equivalences will be the chain homotopy equivalences. We shall denote this category $C(\mathcal{U})$.

4.1. $C(\mathcal{U})$ has a cylinder functor.

Given $f: U \rightarrow V$ a morphism, let $T(f)$ be the chain complex $(T(f))_{p}=U_{p} \oplus$ $U_{p-1} \oplus V_{p}$ with boundary

$$
d_{p} \equiv\left(\begin{array}{ccc}
d_{U} & -1 & 0 \\
0 & -d_{U} & 0 \\
0 & f & d_{V}
\end{array}\right)
$$

We have the following diagram:

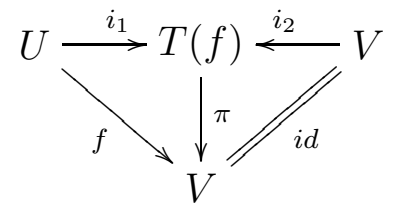

where $j_{1}$ and $j_{2}$ are the obvious inclusions as direct summands. Degree-wise $\pi$ is defined as:

$$
\pi_{p} \equiv\left(f,(-1)^{p-1} f, 1\right)
$$


It is easy to check that Cyl 1 and Cyl 2 are satisfied. The cylinder axiom also holds. To see this, we need to show that $\pi$ is a weak equivalence, i.e. a chain homotopy equivalence. The homotopy inverse is the natural inclusion

$$
i_{2}=\left(\begin{array}{l}
0 \\
0 \\
1
\end{array}\right)
$$

Degree-wise, all is given by the following matrices:

$$
\pi_{p}=\left(f,(-1)^{p-1} f, 1\right) \quad d_{p}=\left(\begin{array}{ccc}
d & -1 & 0 \\
0 & -d & 0 \\
0 & f & d
\end{array}\right) \quad \Gamma_{p}=\left(\begin{array}{ccc}
0 & 0 & 0 \\
1 & 0 & 0 \\
0 & 0 & (-1)^{p}
\end{array}\right)
$$

It is easy to check now that $\Gamma_{p} d_{p+1}+d_{p+2} \Gamma_{p+1}=i_{2} \pi_{p+1}-1$ and $\pi i_{2}=1$.

4.2. $C(\mathcal{U})$ satisfies the saturation and the extension axiom as well.

This is proved by elementary chain complex manipulations involving iterated mapping cones.

When $C(\mathcal{U})$ is given as weak equivalences the chain maps, that induce homotopy equivalence in $C(\mathcal{U} / \mathcal{A})$, we denote it by $C(\mathcal{U}(\mathcal{A})$ ). Obviously the subcategory of chain complexes concentrated in degree 0 is exactly $\mathcal{U}(\mathcal{A}) . C(\mathcal{U} / \mathcal{A})$ has a cylinder functor inherited from $C(\mathcal{U})$ satifying the cylinder axiom, saturation axiom and extension axiom. This follows by working in $C(\mathcal{U} / \mathcal{A})$.

At this point, we can properly apply the generic fibration lemma 2.10 to the functor induced by the identity

$$
C(\mathcal{U}) \longrightarrow C(\mathcal{U}(\mathcal{A}))
$$

obtaining $C(\mathcal{U})^{w}$ as fiber where $w$ denote the weak equivalences in $C(\mathcal{U}(\mathcal{A}))$.

First we would like to show this setting is essentially the one we wanted originally, without chain complexes. In order to do this, we need to introduce the concept of idempotent complete category and restricted idempotent completion.

\section{IDEMPOTENT COMPLETIONS.}

The idempotent completion of an additive category $\mathcal{A}$, denoted $\mathcal{A}^{\wedge}$, is the additive category with objects $(A, p)$ with $p=p^{2}: A \rightarrow A$ and morphisms $f:(A, p) \rightarrow(B, q)$ satisfying $f=q f p: A \rightarrow B$. The identity morphism of $(A, p)$ in $\mathcal{A}^{\wedge}$ is

$$
1_{(A, p)}=p:(A, p) \rightarrow(A, p)
$$

The embedding of additive categories:

$$
\mathcal{A} \hookrightarrow \mathcal{A}^{\wedge}
$$


sending $A$ to $(A, 1)$ is full and cofinal. The morphisms $f:(A, 1) \rightarrow(B, 1)$ in $\mathcal{A}^{\wedge}$ are precisely those in $\mathcal{A}$, and for every $(A, p)$ in $\mathcal{A}^{\wedge}$

$$
(A, p) \oplus(A, 1-p) \underset{\left(\begin{array}{c}
p \\
1-p
\end{array}\right)}{\stackrel{(p, 1-p)}{\rightleftarrows}}(A, 1)
$$

are isomorphisms expressing $(A, p)$ as a direct summand of $(A, 1)$. By the cofinality theorem 2.9 we have a fibration up to homotopy:

$$
K(w S . \mathcal{A}) \rightarrow K\left(w S . \mathcal{A}^{\wedge}\right) \rightarrow B \pi
$$

where $\pi=K_{0}\left(\mathcal{A}^{\wedge}\right) / K_{0}(\mathcal{A})$. In particular, this implies

$$
K_{0}(\mathcal{A}) \longrightarrow K_{0}\left(\mathcal{A}^{\wedge}\right)
$$

$\mathcal{A}^{\wedge}$ has the following properties:

$(P):$ If $f: E \rightarrow F$ is a map, and there is a map $s: F \rightarrow E$ such that splits $f s=1_{F}$, then $f$ is an admissible epimorphism.

And its dual:

$\left(P^{\star}\right):$ If $g: F \rightarrow E$ has $t: E \rightarrow F$ such that $t g=1_{F}$ then $g$ is an admissible monomorphism.

$(P)$ and $\left(P^{\star}\right)$ imply each other. To see more about this we refer the reader to $[11$, 1.11.10] on page 283 .

5.1. It is easy to see that $(P)$ holds for $\mathcal{A}^{\wedge}$.

Let

$$
(U, p) \underset{s}{\stackrel{r}{\rightleftarrows}}(V, q)
$$

be such that $r s=q$. We have also

$$
q r p=r \quad p^{2}=p \quad p s q=s \quad s^{2}=s .
$$

Now $(s r)(s r)=s(r s) r=s q r=(p s q) q r=p s q^{2} r=p s q r=(p s q) r=s r$ so $(U, s r)$ makes sense in $\mathcal{A}^{\wedge}$ and moreover it is an idempotent for $(U, p)$. Since $\mathcal{A}^{\wedge}$ is complete by definition we have

$$
(U, p) \cong(U, p-s r) \oplus(U, s r)
$$


Moreover

$$
(U, s r) \underset{s}{\stackrel{r}{\rightleftarrows}}(V, q)
$$

are isomorphic by those morphisms and therefore $r$ in 5.2 is an admissible epimorphism.

5.3. The isomorphism 5.2.1 is true by the following argument.

If $q:(U, p) \rightarrow(U, p)$ is such that $q^{2}=q$, also $p q p=q$, then

$$
(p q p)(p q p)=(p q p)
$$

thus by the properties of the idempotent completion we have

$$
(U, p) \cong(U, p q p) \oplus(U, p-p q p)
$$

where the isomorphisms are given by the matrices

$$
\left(\begin{array}{c}
p q p \\
p-p q p
\end{array}\right) \quad \text { and } \quad(p q p, p-p q p)
$$

Actually the category $\mathcal{A}^{\wedge}$ satisfies a stronger property than $(P)$, and its dual $\left(P^{\star}\right)$. $\mathcal{A}^{\wedge}$ is Karoubian. We say that an additive category $E$ is Karoubian if whenever $p: E \rightarrow E$ such that $p^{2}=p$ then there is an isomorphism $E \cong E^{\prime} \oplus E^{\prime \prime}$ under which $p$ corresponds to the endomorphism $1 \oplus 0$. In other words it is idempotent complete. See this on page 398 in [11, appendix A] .

Now let $\mathcal{U}$ be an additive category and $\mathcal{A}$ a full subcategory of $\mathcal{U}$.

Definition 5.4. Let $K \subset K_{0}\left(\mathcal{A}^{\wedge}\right)$ be the inverse image of $K_{0}(\mathcal{U})$ under the map $K_{0}\left(\mathcal{A}^{\wedge}\right) \rightarrow K_{0}\left(\mathcal{U}^{\wedge}\right)$. We shall denote the full subcategory of $\mathcal{U}^{\wedge}$ with objects $U \oplus$ $(A, p)$, where $[(A, p)] \in K$ by $\mathcal{U}^{\wedge K}$. Notice that $\mathcal{A}^{\wedge}$ is embedded in $\mathcal{U}^{\wedge}, \mathcal{A}^{\wedge} \hookrightarrow \mathcal{U}^{\wedge}$.

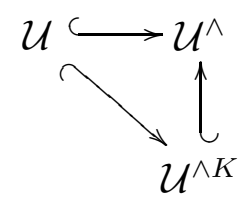

$\mathcal{U}$ is cofinal in $\mathcal{U}^{\wedge K}$ and in $\mathcal{U}^{\wedge}$ hence $\mathcal{U}^{\wedge K}$ is cofinal in $\mathcal{U}^{\wedge}$. We thus obtain a diagram of monomorphisms

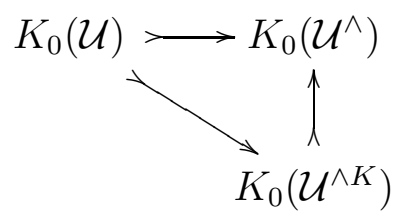


where the images of $K_{0}(\mathcal{U})$ and $K_{0}\left(\mathcal{U}^{\wedge K}\right)$ in $K_{0}\left(\mathcal{U}^{\wedge}\right)$ are the same. Hence

$$
K_{0}(\mathcal{U}) \longrightarrow K_{0}\left(\mathcal{U}^{\wedge K}\right)
$$

is an isomorphism and therefore $\mathcal{U}$ and $\mathcal{U}^{\wedge K}$ have homotopy equivalent $K$-theories, by the cofinality theorem.

In a more general setting we can give the following definition.

Definition 5.5. Given $\mathcal{U}$ an additive category and $K$ a subgroup of $K_{0}(\mathcal{U})$. Let $\mathcal{U}^{\wedge K}$ be the full subcategory of $\mathcal{U}^{\wedge}$ with objects $(U, p)$ so that its stable isomorphism class lies in $K$. When $K=K_{0}(\mathcal{U})$ we denote $\mathcal{U}^{\wedge K_{0}(\mathcal{U})}$ as $\overline{\mathcal{U}}$.

\section{Remark 5.6.}

(i) The category $\overline{\mathcal{U}}$ can be seen in terms of the first definition as $\mathcal{U}^{\wedge K_{0}(\mathcal{U})}$ by taking the trivial filtration $\mathcal{A}=\mathcal{U}$.

(ii) There will be no confusion because of the notation for $\mathcal{U}^{\wedge K}$ in the two definitions above, since in one situation $K \subset K_{0}\left(\mathcal{A}^{\wedge}\right.$ and in the other $K \subset K_{0}\left(\mathcal{U}^{\wedge}\right)$.

5.7. The category $\mathcal{U}$ is cofinal in $\overline{\mathcal{U}}$ and therefore

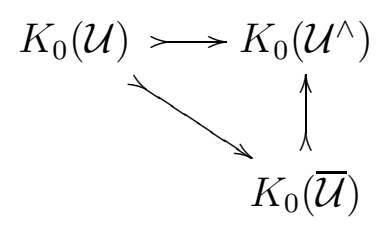

is a commutative diagram where all arrows are monomorphisms. By the same argument as above, $K_{0}(\mathcal{U}) \cong K_{0}(\overline{\mathcal{U}})$ is an isomorphism. Again, by the cofinality theorem $2.9, \mathcal{U}$ and $\overline{\mathcal{U}}$ have homotopy equivalent $K$-theories.

5.8. Note $\overline{\mathcal{U}}$ satisfies the property $(P)$.

We can use an argument similar to the one used in the preceding section for $\mathcal{U}^{\wedge}$. If we have the diagram in $\overline{\mathcal{U}}$

$$
(U, p) \underset{s}{\stackrel{r}{\rightleftarrows}}(V, q)
$$

with $r s=q$, it is also a map in $\mathcal{U}^{\wedge}$ and thus

$$
(U, p) \cong(U, p-s r) \oplus(U, s r)
$$

and

$$
(U, s r) \cong(V, q)
$$

But $(U, p)$ and $(V, q)$ are in $\overline{\mathcal{U}}$ so by the properties of $K_{0}$ and the definition of $\overline{\mathcal{U}}$ we conclude $(U, s r)$ and $(U, p-s r)$ are in $\overline{\mathcal{U}}$. Hence $r$ is an admissible epimorphism and $\overline{\mathcal{U}}$ satisfies the property $(P)$. 
Before we can state and prove the Main Theorem, we need some results on chain complexes.

\section{Chain COMPlex CATEgories}

In this section we give a proof of the theorem due to Thomason and Trobaugh [1] From now on, we will abuse the language as follows. Everything has to be thought of as being passed through the $K$-theory functor. When we say that two categories are homotopy equivalent we mean that their $K$-theories are homotopy equivalent and, any diagram we present must be thought as having the category replaced by its corresponding $K$-theory and every functor replaced by its induced map between the appropriate spaces.

As we have already mentioned, we wish to use the generic fibration lemma, but we can not use it directly because the additive categories do not have cylinder functors. Therefore we replace the categories by their corresponding categories of finite chain complexes.

Proposition 6.1. [11, Theorem 1.11.7] Given $\mathcal{U}$ an additive category that satisfies property $(P)$, let $C(\mathcal{U})$ be its category of finite chain complexes. Assume $\mathcal{U}$ and $C(\mathcal{U})$ are given the usual 'structures' of categories with cofibrations and weak equivalences as we mentioned in sections 3 and 4 Then, the embedding $\mathcal{U} \hookrightarrow C(\mathcal{U})$, as chain complexes of length 1 , induces a homotopy equivalence of $K$-theory spectra.

Proof. It has been shown that $C(\mathcal{U})$ has cylinder functor and satisfies the saturation axiom, the extension axiom and the cylinder axiom, see section 4 . Recall that the weak equivalences in $C(\mathcal{U})$ are the chain homotopy equivalences. We will denote this $(C(\mathcal{U}), w)$. Without changing the subcategory of cofibrations, we can regard $C(\mathcal{U})$ as having as its weak equivalences just the isomorphisms of chain complexes. This 'new structure' on $C(\mathcal{U})$ will be denoted as $(C(\mathcal{U}), i)$. The category $C(\mathcal{U})$ can be thought as $\lim _{\substack{a \rightarrow-\infty \\ b \rightarrow+\infty}} C_{a}^{b}$, where $C_{a}^{b}$ is the full subcategory of complexes in $C(\mathcal{U})$ with $C_{i}=0$ whenever $i \leq a-1$ or $i \geq b+1$. For any $a, b \in \mathbb{Z}, w C_{a}^{b}$ is a category with cofibrations and weak equivalences inherited from $(C(\mathcal{U}), w)$. The category $i C_{a}^{b}$ is the category $C_{a}^{b}$ where the structures are inherited from $(C(\mathcal{U}), i)$ instead. Then we identify $\mathcal{U}$ with $C_{0}^{0}$. It is clear that $w C_{0}^{0}=i C_{0}^{0}$.

In this context, we may consider $\left(C(\mathcal{U})^{w}, i\right)$, which is the full subcategory of $(C(\mathcal{U}), i)$ whose objects are those chain complexes that are contractible in the 'w'sense, i.e. as objects in $(C(\mathcal{U}), w)$. Now, by the generic fibration lemma:

$$
\left(C(\mathcal{U})^{w}, i\right) \rightarrow(C(\mathcal{U}), i) \rightarrow(C(\mathcal{U}), w)
$$

is a fibration, up to homotopy. 
We shall show that $\mathcal{U}(=(\mathcal{U}, w)=(\mathcal{U}, i))$, considering $\mathcal{U}$ embedded in $C(\mathcal{U})$, is the cofiber of the map

$$
\left(C(\mathcal{U})^{w}, i\right) \rightarrow(C(\mathcal{U}), i)
$$

Consider the exact functor

$$
\begin{gathered}
i C_{a}^{b} \longrightarrow \prod_{\#}^{b-a+1} \mathcal{U} \\
C_{\#} \mapsto\left(C_{a}, \ldots, C_{b}\right)
\end{gathered}
$$

We claim it induces a homotopy equivalence of $K$-theories.

For $a=b$, it is clear. Now, by induction, we will show

$$
\begin{aligned}
i C_{a}^{b} & \longrightarrow i C_{a+1}^{b} \times \mathcal{U} \\
\left(C_{a} \rightarrow C_{a+1} \rightarrow \cdots \rightarrow C_{b}\right) & \mapsto\left(\left(C_{a+1} \rightarrow \cdots \rightarrow C_{b}\right),\left(C_{a} \rightarrow 0 \rightarrow \cdots \rightarrow 0\right)\right)
\end{aligned}
$$

induces homotopy equivalence in $K$-theory. This is clear by the additivity theorem 2.7 , since

$$
i C_{a}^{b}=E\left(i C_{a+1}^{b}, C_{a}^{b}, \mathcal{U}\right)
$$

Now, we claim that $i C_{a}^{b^{w}}$ is homotopy equivalent in $K$-theory to $\prod^{b-a} \mathcal{U}$. We do this by induction on the integers $a \leq b$.

For $a=b, i C_{a}^{a^{w}}=i(\mathcal{U})^{w}$ which is equivalent to the 0-category.

For $a=b-1$, it is also clear that

$$
i C_{b-1}^{b^{w}} \equiv\left\{\text { category of complexes } C_{b-1} \stackrel{\partial}{\rightarrow} C_{b} \text { where } \partial \text { is an isomorphism }\right\}
$$

so it is equivalent to $\mathcal{U}$.

We continue by induction on $b-a$. We shall produce a homotopy equivalence:

$$
K\left(i C_{a}^{b^{w}}\right) \stackrel{\cong}{\rightrightarrows} K\left(i C_{a}^{b-1^{w}}\right) \times K\left(i C_{b-1}^{b^{w}} \cong \mathcal{U}\right)
$$

This is obtained by applying the additivity theorem 2.7 to the equivalence of categories

$$
i C_{a}^{b^{w}}=E\left(i C_{a}^{b-1^{w}}, i C_{a}^{b^{w}}, i C_{b-1}^{b^{w}}\right)
$$

We need to show this equality. Given a chain complex $C_{\#}$ in $i C_{a}^{b^{w}}$ we should produce an associated extension with a chain complex of length $b-1$ or less, $\tau^{\leq b-1}\left(C_{\#}\right)$, and other one of length $b, \tau^{b}\left(C_{\#}\right)$. The inverse equivalence of categories takes the total complex $C_{\#}$ and forgets the extensions. It is easy to check that both the equivalence of categories and its inverse are exact functors. 
Since

$$
C_{\#} \equiv\left\{0 \rightarrow C_{a} \rightarrow C_{a+1} \rightarrow \cdots \rightarrow C_{b-2} \stackrel{d_{b-2}}{\longrightarrow} C_{b-1} \stackrel{d_{b-1}}{\longrightarrow} C_{b} \rightarrow 0\right\}
$$

is contractible, we have a chain map $s$ such that $s d+d s=1$. In case degree $b-1$, $s$ is an splitting. Therefore, $d_{b-1}$ is an splitting epimorphism in $\mathcal{U}$. But $\mathcal{U}$ satisfies $(P)$, so there exist $Z_{b-1}$ and an isomorphism such that $C_{b-1} \cong Z_{b-1} \oplus C_{b}$. Moreover, through this isomorphism, $d_{b-1}$ becomes a projection onto $C_{b}$. The maps $d_{b-2}$ and $s$ factor through $Z_{b-1}$. In this way, we obtain shorter contractible chain complexes:

$$
\begin{aligned}
\tau^{\leq b-1}\left(C_{\#}\right) & \equiv\left(0 \rightarrow C_{a} \rightarrow C_{a+1} \rightarrow \cdots \rightarrow C_{b-2} \rightarrow Z_{b-1} \rightarrow 0\right) \\
\tau^{b}\left(C_{\#}\right) & \equiv\left(0 \rightarrow C_{b} \stackrel{\text { id }}{\rightarrow} C_{b} \rightarrow 0\right)
\end{aligned}
$$

Now, $C_{\#}$ fits into the sequence:

$$
\tau^{\leq b-1}\left(C_{\#}\right) \longrightarrow C_{\#} \longrightarrow \tau^{b}\left(C_{\#}\right)
$$

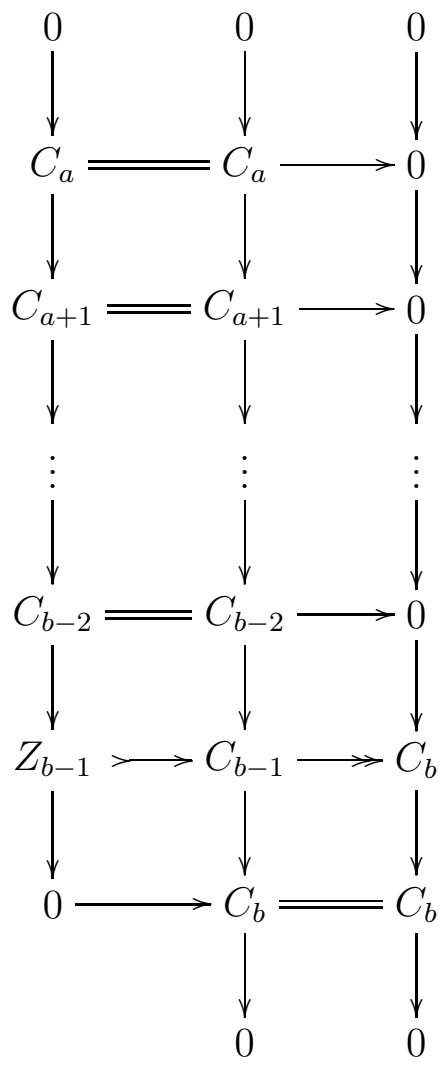

We have the equality of the formula 6.1 .3 as we wanted. The additivity theorem 2.7 can be applied obtaining the homotopy equivalence of $K$-theories we wanted. 
In this proof, we have shown that for $C_{\#}$ in $C_{a}^{b^{w}}, Z_{i} a+1 \leq i \leq b$ are in $\mathcal{U}$ and the functor $C_{\#} \longrightarrow Z_{i}$ is an exact functor, for each $i$. In fact, the homotopy equivalence is induced by

$$
\begin{aligned}
& i C_{a}^{b^{w}} \longrightarrow \quad \stackrel{b-a}{\longrightarrow} \mathcal{U} \\
& C_{\#} \longrightarrow\left(Z_{a+1}, \ldots, Z_{b}\right)
\end{aligned}
$$

Now, let us consider the exact inclusion

$$
i C_{a}^{b^{w}} \longrightarrow i C_{a}^{b}
$$

and the induced maps of $K$-theory spectra:

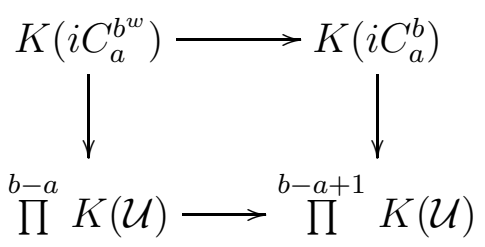

Given a chain complex $C_{\#}$ in $i C_{a}^{b^{w}}$ the term in $\prod^{b-a} \mathcal{U}$ is $\left(Z_{a+1}, \ldots, Z_{b}\right)$ and in $\prod^{b-a+1} \mathcal{U}$ is $\left(C_{a}, C_{a+1}, \ldots, C_{b}\right)$. Since in $C_{\#}$ we can identify, for each dimension, the exact sequence

$$
Z_{k} \longrightarrow C_{k} \longrightarrow Z_{k+1}
$$

It can be said, using the additivity theorem 2.7, that the map, once passing to $K$ theory, sending $C_{\#}$ to $C_{k}$ is homotopic to the 'sum' of the maps sending $C_{\#}$ to $Z_{k}$ and $C_{\#}$ to $Z_{k+1}$.

Therefore, we can assume the map in the above square

$$
\prod^{b-a} K(\mathcal{U}) \longrightarrow \prod^{b-a+1} K(\mathcal{U})
$$

is induced by

$$
\left(Z_{a+1}, \ldots, Z_{b}\right) \longrightarrow\left(Z_{a+1}, Z_{a+1} \oplus Z_{a+2}, \ldots, Z_{b-1} \oplus Z_{b}, Z_{b}\right)
$$

The homotopy cofiber of this map is $K(\mathcal{U})$. It is induced by

$$
\begin{aligned}
\prod^{b-a+1} K(\mathcal{U}) & \rightarrow K(\mathcal{U}) \\
\left(x_{a}, \ldots, x_{b}\right) & \rightarrow \sum_{k=a}^{b}(-1)^{k} x_{k}
\end{aligned}
$$


Taking direct limits $(a \rightarrow-\infty, b \rightarrow+\infty)$, we get the cofiber homotopy sequence

$$
\begin{aligned}
K\left(i C^{w}\right) \longrightarrow K(i C) & \longrightarrow K(\mathcal{U}) \\
C_{\#} & \longrightarrow \sum(-1)^{k} C_{k}
\end{aligned}
$$

By the generic fibration lemma 2.10, the homotopy cofiber spectrum is, up to homotopy, $K(w C)$. Thus, there is a homotopy equivalence

$$
K(\mathcal{U}) \stackrel{\cong}{\rightrightarrows} K(w C)
$$

induced by the exact functor $\mathcal{U} \rightarrow C(\mathcal{U})$.

Corollary 6.2. Given an additive category $\mathcal{U}$ we have

$$
K(\mathcal{U}) \simeq K(C(\mathcal{U}))
$$

induced by the inclusion of categories.

Proof. If $\mathcal{U}$ does not satisfy the property $(P)$ then $\overline{\mathcal{U}}$ does, see section 5. Moreover, $\mathcal{U}$ is cofinal in $\overline{\mathcal{U}}$ and both have the 'same' $K$-theory, see section 5 . Chasing the following diagram we obtain the result.

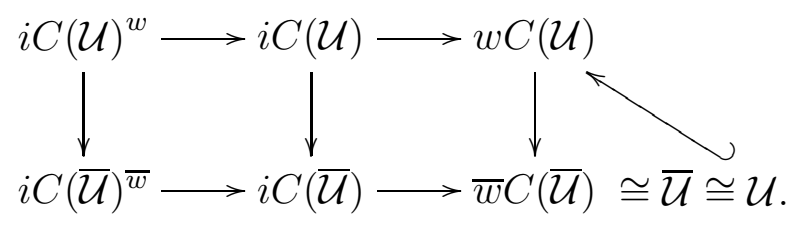

The top and bottom row are fibrations by the generic fibration lemma 2.10. The two isomorphisms at the bottom are consequences of proposition 6.1 and cofinality 2.9. The vertical arrows on the left and on the middle are homotopy equivalences because $\mathcal{U}$ is cofinal in $\overline{\mathcal{U}}$. We can conclude that the vertical arrow on the right is a homotopy equivalence by considering the long exact sequences of homotopy groups corresponding to the two fibrations generated by the rows. The right hand side diagram commutes and therefore the result is obtained.

\section{Proof of the Main theorem}

In this section we will prove

Theorem 7.1. [7, Theorem 5.3] Given $\mathcal{U}$, an additive $\mathcal{A}$-filtered category, then

$$
\mathcal{A}^{\wedge K} \rightarrow \mathcal{U} \rightarrow \mathcal{U} / \mathcal{A}
$$

is a fibration, up to homotopy. Here $K$ is the inverse image of $K_{0}(\mathcal{U}) \subset K\left(\mathcal{U}^{\wedge}\right)$ under the induced map $K\left(\mathcal{A}^{\wedge}\right) \rightarrow K\left(\mathcal{U}^{\wedge}\right)$. 
We will now apply the generic fibration lemma to the functor

$$
C(\mathcal{U}) \rightarrow C(\mathcal{U}(\mathcal{A}))
$$

and show that $C(\mathcal{U}(\mathcal{A}))$ and $C(\mathcal{U} / \mathcal{A})$ are homotopy equivalent in K-theory. By corollary 6.2 we thus have models for $K(\mathcal{U})$ and $K(\mathcal{U} / \mathcal{A})$.

Proposition 7.2. Let $\mathcal{U}$ be an additive $\mathcal{A}$-filtered category. The functor

$$
F: C(\mathcal{U}(A)) \rightarrow C(\mathcal{U} / \mathcal{A})
$$

which is the identity on the objects and takes classes on the morphisms mod\{cc maps $\}$, induces a homotopy equivalence of $K$-theories.

Proof. We will use the approximation theorem 2.12. The weak equivalences $w C(\mathcal{U}(A))$ and $w C(\mathcal{U} / \mathcal{A})$ satisfy the saturation axiom. $C(\mathcal{U}(A))$ has a natural cylinder functor inherited from $C(\mathcal{U})$ satisfying the cylinder axiom, see section 4 .

App 1: is satisfied trivially, because of the definition of $w C(\mathcal{U}(A))$.

$\overline{A p p ~ 2}$ : will be easy after the following remark:

By the properties of the $\mathcal{A}$-filtration of $\mathcal{U}$ any $D_{\#}$ in $C(\mathcal{U} / \mathcal{A})$ is isomorphic in $\mathcal{U} / \mathcal{A}$ to a chain complex from $C(\mathcal{U})$ [4, Proof of theorem 4.1].

Let $C_{\#}$ be in $C(\mathcal{U}(A))$ and $F\left(C_{\#}\right) \stackrel{x}{\rightarrow} D_{\#}$ in $C(\mathcal{U} / \mathcal{A})$. We are assuming, by the remark, that $D_{\#}$ is isomorphic by $\varphi$ to a chain complex $D_{\#}^{\prime}$ which is from $C(\mathcal{U})$. We can apply the cylinder functor to $\varphi x$, obtaining an object, $T(\varphi x)$ in $C(\mathcal{U})$. The diagram is

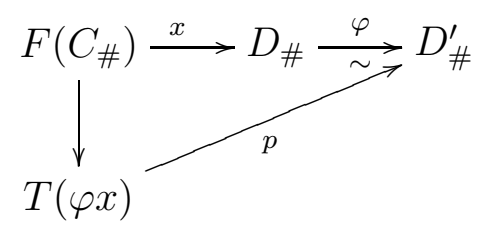

where $\varphi$ is an isomorphism and hence a weak equivalence. So is $p$ by the cylinder axiom. Therefore $\varphi^{-1} p$ is a weak equivalence. All of this only needs to commute $\bmod \mathcal{A}$ because the ambient category is $\mathcal{U} / \mathcal{A}$.

Therefore $F$ verifies the approximation properties, and by 2.12 , it induces a homotopy equivalence of $K$-theories.

This last result has told us we are on the right track. Therefore our next step is to investigate $C(\mathcal{U})^{w}$, the fiber of $C(\mathcal{U}) \longrightarrow C(\mathcal{U}(\mathcal{A}))$. We need the following two results from [8] (see also [4]) in order to continue the argumentat.

Proposition 7.3. [4, Proposition 4.7] Let $\mathcal{U}$ be an $\mathcal{A}$-filtered category. A chain complex $U_{\#}$ in $\mathcal{U}$ is $\mathcal{A}$-dominated iff the induced $\mathcal{U} / \mathcal{A}$-chain complex is contractible. 
Lemma 7.4. [4, Lemma 4.8] Let $\mathcal{A}$ be a full subcategory of $\mathcal{U}, U_{\#}$ an $\mathcal{A}$-dominated chain complex in $\mathcal{U}$. Let $K$ be the inverse image of $K_{0}(\mathcal{U})$ under the induced map $K_{0}\left(\mathcal{A}^{\wedge}\right) \longrightarrow K_{0}\left(\mathcal{U}^{\wedge}\right)$, and let $\mathcal{U}^{\wedge K}$ be the full subcategory with objects $U \oplus(A, p)$, $[(A, p)] \in K$.

Then the induced chain complex in $\mathcal{U}^{\wedge K}$ under the inclusion $\mathcal{U} \rightarrow \mathcal{U}^{\wedge K}$ is chain homotopy equivalent to a chain complex in $\mathcal{A}^{\wedge K}$.

In order to apply 7.3 we restate it in the following way.

Proposition 7.5. Let $\mathcal{U}$ be an $\mathcal{A}$-filtered additive category and $C(\mathcal{U})$ its category of finite chain complexes. Let $C(\mathcal{U})^{w}$ be the full subcategory of chain complexes in $\mathcal{U}$ that are contractible in $\mathcal{U} / \mathcal{A}$ and let $C(\mathcal{U})^{\mathcal{A}}$ be the full subcategory of chain complexes in $\mathcal{U}$ that are $\mathcal{A}$-dominated. Then $C(\mathcal{U})^{w}=C(\mathcal{U})^{\mathcal{A}}$.

The category $\mathcal{U}^{\wedge K} / \mathcal{A}^{\wedge K}$ is clearly equivalent to $\mathcal{U} / \mathcal{A}$ and $\mathcal{U}$ is cofinal in $\mathcal{U}^{\wedge K}$, see section 5 . Therefore the functor induced by this cofinality

$$
C(\mathcal{U} / \mathcal{A}) \longrightarrow C\left(\mathcal{U}^{\wedge K} / \mathcal{A}^{\wedge K}\right)
$$

induces a homotopy equivalence. Also the functor

$$
C(\mathcal{U}) \longrightarrow C\left(\mathcal{U}^{\wedge K}\right)
$$

induces a homotopy equivalence of $K$-theories and both $K$-theories are homotopy equivalent to that of $\mathcal{U}$ through the respective inclusions.

Let us denote by $w^{\prime}$ the weak equivalences in $C\left(\mathcal{U}^{\wedge K}\left(\mathcal{A}^{\wedge K}\right)\right)$. If we apply the generic fibration lemma 2.10 , to

$$
C\left(\mathcal{U}^{\wedge K}\right) \longrightarrow C\left(\mathcal{U}^{\wedge K}\left(\mathcal{A}^{\wedge K}\right)\right)
$$

we obtain this fibration, up to homotopy:

$$
C\left(\mathcal{U}^{\wedge K}\right)^{w^{\prime}} \longrightarrow C\left(\mathcal{U}^{\wedge K}\right) \longrightarrow C\left(\mathcal{U}^{\wedge K}\left(\mathcal{A}^{\wedge K}\right)\right)
$$

as we did for $C(\mathcal{U})$ in the proof of proposition 7.2. On the other hand, applying the generic fibration lemma 2.10 to

$$
C(\mathcal{U}) \longrightarrow C(\mathcal{U}(\mathcal{A}))
$$

we obtain

$$
C(\mathcal{U})^{w} \longrightarrow C(\mathcal{U}) \longrightarrow C(\mathcal{U}(\mathcal{A}))
$$

The cofinality gives us maps to compare both sequences which with 7.2 produces the 
following diagram.

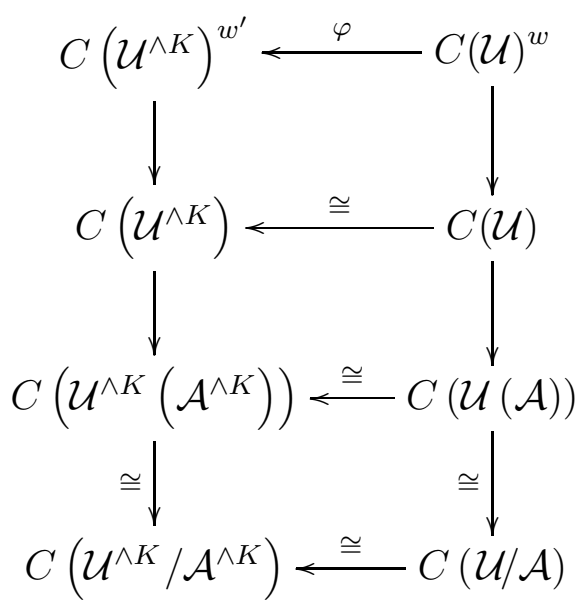

So, by observing the long exact sequence of homotopy groups we can conclude that Proposition 7.6. The induced functor $\varphi: C(\mathcal{U})^{w} \longrightarrow C\left(\mathcal{U}^{\wedge K}\right)^{w^{\prime}}$ induces a homotopy equivalence of $K$-theories.

We have the natural inclusion $F: C\left(\mathcal{A}^{\wedge K}\right) \longrightarrow C\left(\mathcal{U}^{\wedge K}\right)^{w^{\prime}}$ and by proposition 7.5 ( or more exactly $\left[4\right.$, Proposition 4.7]) we have $C(\mathcal{U})^{\mathcal{A}}=C(\mathcal{U})^{w}$ and $C\left(\mathcal{U}^{\wedge K}\right)^{\mathcal{A}^{\wedge K}}=$ $C\left(\mathcal{U}^{\wedge K}\right)^{w^{\prime}}$. The diagram above becomes then

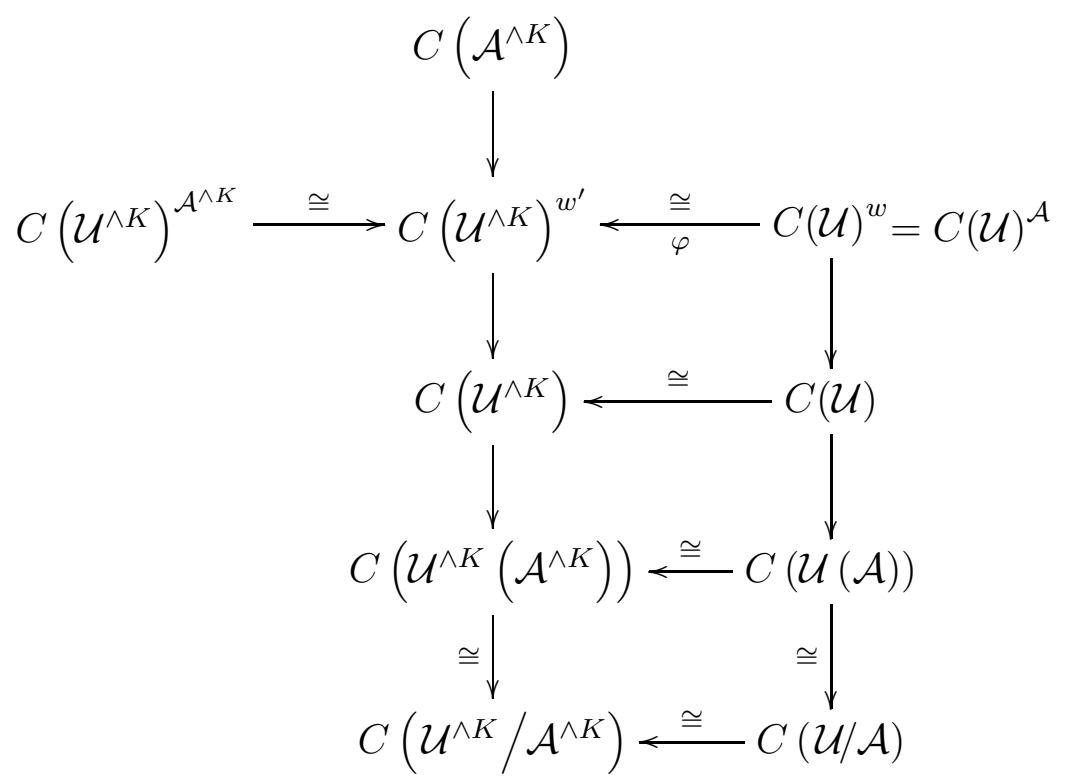


Proposition 7.7. The functor $F: C\left(\mathcal{A}^{\wedge K}\right) \longrightarrow C\left(\mathcal{U}^{\wedge K}\right)^{w^{\prime}}$ induces a homotopy equivalence.

Proof. We want to apply the approximation theorem 2.12 to $F$. The categories $C\left(\mathcal{A}^{\wedge K}\right)$ and $C\left(\mathcal{U}^{\wedge K}\right)^{w^{\prime}}$ satisfy the saturation axiom. $C\left(\mathcal{A}^{\wedge K}\right)$ satisfies the cylinder axiom as well. Let us check App 1 and App 2.

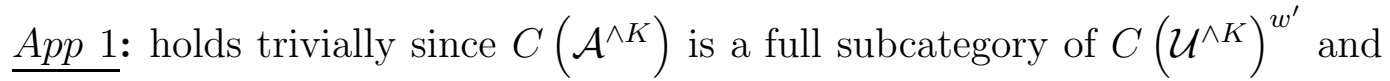
therefore inherits weak equivalences from $C\left(\mathcal{U}^{\wedge K}\right)$.

App 2: follows easierly after the following remark:

Given $B_{\#}$ in $C\left(\mathcal{U}^{\wedge K}\right)^{w^{\prime}}$ then $B_{\#}$ is $\mathcal{U}^{\wedge K} / \mathcal{A}^{\wedge K}$-contractible. But any chain complex in $C\left(\mathcal{U}^{\wedge K}\right)$ is homotopy equivalent to one in $C(\mathcal{U})$. See the proof of $\left[4\right.$, Theorem 4.1]. Therefore $B_{\#}$ is homotopy equivalent to $B_{\#}^{\prime}$ in $C(\mathcal{U}) \subset$ $C\left(\mathcal{U}^{\wedge K}\right)$. But $\mathcal{U}^{\wedge K} / \mathcal{A}^{\wedge K}=\mathcal{U} / \mathcal{A}$. This means that $B_{\#}^{\prime}$ is $\mathcal{U} / \mathcal{A}$-contractible. Then by $7.5 B_{\#}^{\prime}$ is $\mathcal{A}$-dominated hence by lemma $7.4 B_{\#}^{\prime}$ is homotopy equivalent, in $\mathcal{U}^{\wedge K}$, to a chain complex $A_{\#}^{\prime}$ in $\mathcal{A}^{\wedge K}$. We conclude that $B_{\#}$ is homotopy equivalent to an object $A_{\#}^{\prime}$ in $\mathcal{A}^{\wedge K}$.

Now, let us try to verify App 2.

Let $A_{\#} \stackrel{f}{\rightarrow} B_{\#}$ be a morphism from an object in $C\left(\mathcal{A}^{\wedge K}\right)$ to an object in $C\left(\mathcal{U}^{\wedge K}\right)^{w^{\prime}}$. By the remark above, we have the homotopy equivalence $i: B_{\#} \stackrel{\sim}{\rightarrow} A_{\#}^{\prime}$ with inverse $r$ such that $\partial \Gamma+\Gamma \partial=r i-1$ where $\Gamma$ is a chain homotopy. The composite $A_{\#} \stackrel{f}{\rightarrow} B_{\#} \stackrel{i}{\rightarrow} A_{\#}^{\prime}$ lies in $C\left(\mathcal{A}^{\wedge K}\right)$. We can apply the cylinder functor to it.

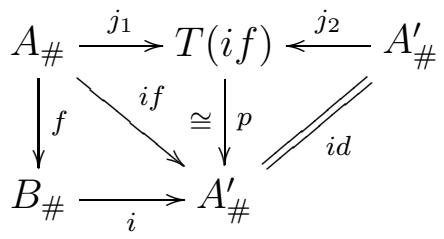

It is left to define $f^{\prime}: T(i f) \longrightarrow B_{\#}$ such that $f^{\prime} j_{1}=f$ and $f^{\prime}$ is a weak equivalence. We define $f^{\prime}$ as follows.

$$
(T(i f))_{p}=A_{p} \oplus A_{p-1} \oplus A_{p}^{\prime} \stackrel{f_{p}^{\prime}}{\longrightarrow} B_{p} \quad f_{p}^{\prime}=(f, \Gamma f, r)
$$

Let us check $f^{\prime}$ is a chain map.

Since

$$
r \partial=\partial r, f \partial=\partial f \quad \text { and } \quad \partial \Gamma+\Gamma \partial=r i-1
$$

then

$$
\partial \Gamma=-1-\Gamma \partial+r i
$$


and hence

$$
\begin{aligned}
f^{\prime} d=(f, \Gamma f, r) \cdot\left(\begin{array}{ccc}
\partial & -1 & 0 \\
0 & -\partial & 0 \\
0 & i f & \partial
\end{array}\right) & =(f \partial,-f-\Gamma f \partial+r i f, r \partial) \\
& =(\partial f, \partial \Gamma f, \partial r)=\partial f^{\prime}
\end{aligned}
$$

A chain homotopy inverse for $f^{\prime}$ is $\left(\begin{array}{l}0 \\ 0 \\ i\end{array}\right)=j_{2} i$.

Now,

$$
(f, \Gamma f, r) \cdot\left(\begin{array}{l}
0 \\
0 \\
i
\end{array}\right)=r i
$$

$\left(\begin{array}{l}0 \\ 0 \\ i\end{array}\right)$ is a weak equivalence, since $j_{2}$ and $i$ are. Then, by saturation, since $r i$ is a weak equivalence, so is $f^{\prime}$. Clearly $f^{\prime} j_{1}=f$. So we have

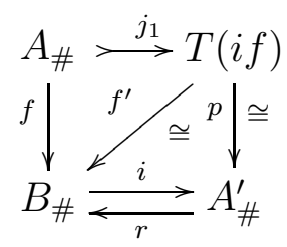

We have verified App 2, and get the result.

Corollary 7.8. $C\left(\mathcal{A}^{\wedge K}\right)$ is homotopy equivalent to $C(\mathcal{U})^{\mathcal{A}}$.

Proof. By proposition $7.7 C\left(\mathcal{A}^{\wedge K}\right)$ is homotopy equivalent to $C\left(\mathcal{U}^{\wedge K}\right)^{w^{\prime}}$, but this is homotopy equivalent to $C(\mathcal{U})^{w}$ by proposition 7.6 , which by proposition 7.5 is h.e. to $C(\mathcal{U})^{\mathcal{A}}$.

Proof of Main Theorem. Let us condense all of the above as follows.

Applying the generic fibration lemma 2.10 to

$$
C(\mathcal{U}) \longrightarrow C(\mathcal{U}(\mathcal{A}))
$$

we obtain the fibration

$$
C(\mathcal{U})^{w} \longrightarrow C(\mathcal{U}) \longrightarrow C(\mathcal{U}(\mathcal{A}))
$$


but by proposition $7.2, C(\mathcal{U}(\mathcal{A})) \simeq C(\mathcal{U} / \mathcal{A})$ and by proposition $7.5, C(\mathcal{U})^{w} \cong$ $C(\mathcal{U})^{\mathcal{A}}$. So, 7.8 .1 now looks like

$$
C(\mathcal{U})^{\mathcal{A}} \longrightarrow C(\mathcal{U}) \longrightarrow C(\mathcal{U} / \mathcal{A}) \text {. }
$$

But by corollary $7.8, C(\mathcal{U})^{\mathcal{A}} \cong C\left(\mathcal{A}^{\wedge K}\right)$, therefore 7.8 .2 becomes

$$
C\left(\mathcal{A}^{\wedge K}\right) \longrightarrow C(\mathcal{U}) \longrightarrow C(\mathcal{U} / \mathcal{A})
$$

Finally, applying corollary 6.2 to the three terms, we obtain that

$$
\mathcal{A}^{\wedge K} \longrightarrow \mathcal{U} \longrightarrow \mathcal{U} / \mathcal{A}
$$

is a fibration, up to homotopy.

If $\mathcal{A}$ is idempotent complete, then $\mathcal{A}$ and $\mathcal{A}^{\wedge K}$ have the same $K$-theory. Therefore

\section{Corollary 7.9.}

$$
\mathcal{A} \rightarrow \mathcal{U} \rightarrow \mathcal{U} / \mathcal{A}
$$

is a fibration, up to homotopy.

We finally show how to use the theorem to obtain excision as in [3] for bounded K-theory. Let $M=M_{1} \cup M_{2}$ be a metric space decomposed as two metric subspaces. Let $\mathcal{U}_{1}=\mathcal{C}(M ; R)$ the category of finitely generated free $R$-modules parameterized by $M$ and bounded morphisms, as in [7]. Let $\mathcal{A}_{1}=\mathcal{C}(M ; R)_{M_{1}}$, the full subcategory with objects having support in a bounded neighborhood of $M_{1}$. Then clearly $\mathcal{U}_{1}$ is $\mathcal{A}_{1}$ filtered, and $\mathcal{C}(M ; R)_{M_{1}} \cong \mathcal{C}\left(M_{1} ; R\right)$. Similarly let $\mathcal{U}_{2}=\mathcal{C}(M ; R)_{M_{2}} \cong \mathcal{C}\left(M_{2} ; R\right)$ and $\mathcal{A}_{2}$ the full subcategory with objects support in a bounded neighborhood of $M_{1}$ intersected with a bounded neighborhood of $M_{2}$. It is easy to see that

$$
\mathcal{U}_{1} / \mathcal{A}_{1} \cong \mathcal{U}_{2} / \mathcal{A}_{2}
$$

and we obtain excision from the diagram

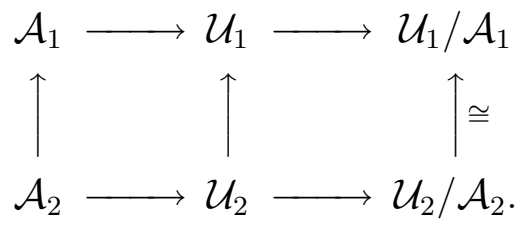

To give a proof of 1.0 .2 we need to recall the definition of $K^{-\infty}$. Let $\mathcal{A}$ be an additive category, $M$ a proper metric space.

Definition 7.10. The bounded category $\mathcal{C}(M ; \mathcal{A})$ has objects $A=\left\{A_{x}\right\}_{x \in M}$, a collection of objects from $\mathcal{A}$ indexed by points of $M$, satisfying $\left\{x \mid A_{x} \neq 0\right\}$ is locally finite in $M$. A morphism $\phi: A \rightarrow B$ is a collection of morphisms $\phi_{y}^{x}: A_{x} \rightarrow B_{y}$ so that there exists $k=k(\phi)$ so $\phi_{y}^{x}=0$ if $d_{M}(x, y)>k$. 
Composition is defined as matrix multiplication. Given a subspace $N \subset M$, we denote the full subcategory with objects $A$ so that $\left\{x \mid A_{x} \neq 0\right\}$ is contained in a bounded neighborhood of $N$ by $\mathcal{C}(M ; \mathcal{A})_{N}$. It is easy to see that $\mathcal{C}(M ; \mathcal{A})$ is $\mathcal{C}(M, \mathcal{A})_{N}$-filtered . We denote the quotient category by $\mathcal{C}(M, \mathcal{A})^{>N}$. We shall need this in the particular case when $M$ is euclidean space $R^{i}$. Consider $\mathcal{C}\left(R^{i} ; \mathcal{A}\right) \rightarrow \mathcal{C}\left(R^{i+1} ; \mathcal{A}\right)$ induced by the standard inclusion. This inclusion factors through $H_{ \pm}^{i+1}$ where $H_{+}^{i+1}$ and $H_{-}^{i+1}$ are the two halfspaces intersecting in $R^{i}$. Clearly $\mathcal{C}\left(H_{ \pm}^{i+1} ; \mathcal{A}\right)$ has an Eilenberg swindle shifting modules by 1 in the direction of the last coordinate, hence these categories have trivial $K$-theory so the map

$$
K\left(\mathcal{C}\left(R^{i} ; \mathcal{A}\right)\right) \rightarrow K\left(\mathcal{C}\left(R^{i+1} ; \mathcal{A}\right)\right)
$$

is canonically null homotopic in two ways thus giving a functorial map

$$
\Sigma K\left(\mathcal{C}\left(R^{i} ; \mathcal{A}\right)\right) \rightarrow K\left(\mathcal{C}\left(R^{i+1} ; \mathcal{A}\right)\right)
$$

or by adjointness

$$
K\left(\mathcal{C}\left(R^{i} ; \mathcal{A}\right)\right) \rightarrow \Omega K\left(\mathcal{C}\left(R^{i+1} ; \mathcal{A}\right)\right)
$$

It follows from 1.0.1 that this is an isomorphism in homotopy groups in dimensions bigger than 0 . We define

$$
K^{-\infty}(\mathcal{A})=\text { hocolim } \Omega^{i} K\left(\mathcal{C}\left(R^{i} ; \mathcal{A}\right) .\right.
$$

It is easy to see that if $\mathcal{U}$ is $\mathcal{A}$-filtered, then $\mathcal{C}\left(R^{i} ; \mathcal{U}\right)$ is $\mathcal{C}\left(R^{i} ; \mathcal{A}\right)$-filtered and we thus recover the fibration of spectra

$$
K^{-\infty}(\mathcal{A}) \rightarrow K^{-\infty}(\mathcal{U}) \rightarrow K^{-\infty}(\mathcal{U} / \mathcal{A})
$$

by taking the homotopy colimit of the fibrations

$$
\Omega^{i} K\left(\mathcal{C}\left(R^{i} ; \mathcal{A}\right)^{K_{i}}\right) \rightarrow \Omega^{i} K\left(\mathcal { C } ( R ^ { i } ; \mathcal { U } ) \rightarrow \Omega ^ { i } K \left(\mathcal{C}\left(R^{i} ; \mathcal{U} / c a\right)\right.\right.
$$

where $K_{i}$ is the appropriate subgroup of $K_{0}\left(\mathcal{C}\left(R^{i} ; \mathcal{A}\right)^{\wedge}\right.$.

\section{REFERENCES}

1. D. A. Anderson and H. J. Munkholm, Foundations of Boundedly Controlled Algebraic and Geometric Topology, Lecture Notes in Mathematics, vol. 1323, Springer-Verlag, Berlin-New York, 1988.

2. D. R. Anderson, F. Connolly, S. C. Ferry, and E. K. Pedersen, Algebraic K-theory with continuous control at infinity., J. Pure Appl. Algebra 94 (1994), 25-47.

3. G. Carlsson, Bounded K-theory and the assembly map in algebraic K-theory, Proceedings of Oberwolfach conference on the Novikov conjecture (S. C. Ferry, J. Rosenberg, and A. A. Ranicki, eds.), (To appear).

4. G. Carlsson and E. K. Pedersen, Controlled algebra and the Novikov conjectures for K- and L-theory, Topology (1993), (To appear).

5. M. Karoubi, Foncteur derivees et K-theorie, Lecture Notes in Mathematics, vol. 136, SpringerVerlag, Berlin-New York, 1970. 
6. Randy McCarthy, On fundamental theorems of algebraic K-theory, Topology 32 (1993), 325328.

7. E. K. Pedersen and C. Weibel, K-theory homology of spaces, Algebraic Topology, Proceedings Arcata 1986 (Berlin-New York) (G. Carlsson, R. L. Cohen, H. R. Miller, and D. C. Ravenel, eds.), Springer-Verlag, 1989, Lecture Notes in Mathematics 1370, pp. 346-361.

8. A. A. Ranicki, Lower $K$ - and L-theory, Lond. Math. Soc. Lect. Notes, vol. 178, Cambridge Univ. Press, Cambridge, 1992.

9. R. Staffeldt, On fundamental theorems on algebraic K-theory, K-theory 1 (1989), 511-532.

10. R. Thomason, First quadrant spectral sequences in algebraic K-theory via homotopy colimits, Comm. in Alg. 10 (1982), 1589-1668.

11. R. Thomason and Trobaugh, Higher algebraic K-theory of schemes and of derived categories, The Grothendieck Festschrift (Boston), Birkhäuser, 1990, pp. 247-435.

12. F. Waldhausen, Algebraic K-theory of generalized free products, Ann. of Math. 108 (1978), $135-256$.

13. Algebraic K-theory of spaces, Algebraic and Geometric Topology, Proceedings Rutgers 1983 (Berlin-New York) (A. Ranicki, N. Levitt, and F. Quinn, eds.), Springer-Verlag, 1985, Lecture Notes in Mathematics 1126.

Dept. of Math. Sci., Binghamton University 